\title{
Research Article \\ SSCM: An Unambiguous Acquisition Algorithm for CBOC Modulated Signal
}

\author{
Yongxin Feng, Shunchao Fei, Fang Liu, and Bo Qian \\ Communication and Network Institute, Shenyang Ligong University, Shenyang 110159, China \\ Correspondence should be addressed to Yongxin Feng; fengyongxin@263.net
}

Received 10 May 2017; Revised 19 August 2017; Accepted 30 August 2017; Published 21 December 2017

Academic Editor: Tho Le-Ngoc

Copyright ( 2017 Yongxin Feng et al. This is an open access article distributed under the Creative Commons Attribution License, which permits unrestricted use, distribution, and reproduction in any medium, provided the original work is properly cited.

\begin{abstract}
Composite binary offset carrier (CBOC) signal has been widely researched in GNSS. The main ingredient of CBOC signal is BOC $(1,1)$ signal. Usually, the acquisition method for $\mathrm{BOC}(1,1)$ signal is used to capture CBOC signal, while the research of special acquisition method for CBOC signal is rare. In this letter, according to the principle and characteristics of CBOC signal, a special side-peak cancellation method (SSCM) is proposed and simulated. In this method, two special auxiliary signals are introduced. And the local reference signals are obtained by multiplying the data channel signal and pilot channel signal by the auxiliary signals. The cross-correlation results from the received pilot signal and the two local pilot signals with different auxiliary signals will subtract from one another. Then, side peaks of correlation function and in-band noise of pilot channel are suppressed, while the data channel has the same operation results. At last the outputs of pilot channel and data channel will be added up to make full use of the received signal power. By this way, the acquisition efficiency, accuracy, and adaptability to low signal-to-noise ratio (SNR) conditions for CBOC signal have been improved, alongside possible solution when the GNSS receiver works in a critical environment.
\end{abstract}

\section{Introduction}

With the development of wireless devices, radio environment is becoming more and more complex. Global Navigation Satellite Systems (GNSS) have to share the crowded frequency source and work in terrible environment with multipath or interference. Then, in 2004, the BOC modulation was proposed by the European Union (EU) and the US, which can be used for modernized civil Global Position System (GPS) signal on L1 band and Galileo Open Service (OS) on E1 band [1]. The new BOC modulation reduces the interference level caused by the existing GPS L1 C/A signal, since it splits the power spectral away from the center frequency. In 2007, in order to improve the interoperability and compatibility between the PRN code tracking accuracy and navigation systems, MBOC modulation has been recommended by the GPS-Galileo working group. Multiplexed Binary Offset Carrier (MBOC) [2] signal is denoted as the optimization modulation method instead of the initial $\operatorname{BOC}(1,1)$ modulation, which can restrain multipath.

The MBOC modulated signal can be produced by $\mathrm{CBOC}$ or time-multiplexed BOC (TMBOC) signals. This new modulation allocates a wide band signal $\operatorname{BOC}(6,1)$ in E1/L1 band without interfering with other existing signals and realizes the compatibility and interoperability between GPS and Galileo system. CBOC modulated signal can get more high-frequency components on the power spectral density (PSD) which improves the performance of tracking accuracy and antimultipath capability. However, some drawbacks have been noted, especially associated with the multiple side peaks of autocorrelation function (ACF) causing the ambiguity problem. In order to remove the side peaks, several acquisition algorithms have been proposed and introduced in the past few years, including BPSK-like technique [3], ASPeCT technique [4], pseudo correlation function (PCF) technique [5-7], subcarrier phase cancellation (SCPC) technique [8], and unambiguous acquisition algorithms without auxiliary signals [9]. Nevertheless, most of the methods are designed for BOC modulated signal. Therefore, a novel acquisition algorithm for the CBOC signal is proposed.

The main contributions of this paper are enumerated below:

(i) An optimized acquisition technique named SSCM is proposed to remove side peaks and then the ambiguity problem can be restrained. 
(ii) CBOC modulated signal is an important part of modern navigation systems. Some acquisition methods only use part of signal power to capture signal, while SSCM make full use of CBOC signal power. Then the acquisition performance is improved.

(iii) In order to overcome the complicated wireless environment, this method is suitable when the GNSS receiver works in a serious condition, which is based on the antijamming performance of modern GNSS signal.

The remainder of this paper is organized as follows. In Section 2, before expound SSCM, some related work has been undertook. In Section 3, properties of the CBOC modulated signal are shown. In Section 4, SSCM with noise reduction for $\mathrm{CBOC}$ modulated signal is presented in detail. Section 5 simulates and compares the proposed algorithm with preexisting acquisition algorithms. Lastly, Section 6 concludes the paper.

\section{Related Work}

Several techniques have been proposed in the last ten years to overcome ambiguity problem. The BPSK-like method regards $\mathrm{BOC}$ or $\mathrm{CBOC}$ modulated signal as the sum of binary phase shift keying (BPSK) signals, using filtering, shifting, and integration to change the ACF shape into BPSK-like correlation function shape. Then, an unambiguous correlation function with a decreasing precision is obtained. However, the structure of BPSK-like method is complex and expensive to implement. The in-band noise of intermediate frequency (IF) signal will have an effect on acquisition. In [4], ASPeCT method has a good performance within solving the ambiguity tracking problem, but also the single peak feature and the inhibiting of side peaks are unsatisfactory. Additionally, the $\operatorname{BOC}(6,1)$ component will be regarded as noise in the ASPeCT method. Then, it is possible that some side peak will be larger than main peak, and false lock will happen. According to literature [5], PCF method uses similar locally BOC modulated signals to obtain unambiguous function. This method shows a good adaptability. However, some disadvantages such as lower main peak and energy loss are unavoidable. The subcarrier phase cancellation (SCPC) technique generates an in-phase local subcarrier signal and a quadra-phase local subcarrier signal, which are used to correlate with the received filtered signal. The outputs of two correlation channels are combined to remove side peaks, and an unambiguous correlation function is obtained. However, the suppression of the in-band noise and the utilization of two channels of CBOC modulated signal have not been fully considered. When the in-band noise is serious and no special care is taken, false acquisition or biased tracking will occur. In order to remove the ambiguities of ACF and unwanted replicas of the signal spectrum, a quick unambiguous acquisition algorithm for BOC modulated signals is proposed [10], which exploits a reduced-complexity filter composed of only seven nonzero samples. However, the high-frequency power is sacrificed when the scheme is applied to the CBOC modulated signal. Thus, the advantages of CBOC modulated signal will be wasted. New unambiguous acquisition algorithms, using auxiliary signals, have been proposed in [10-12]. However, all of them are proposed for sine-BOC or cosine$\mathrm{BOC}$ modulated signal in particular. When considering the unique characteristic of CBOC modulated signals, it is more urgent to study the effective acquisition algorithms for CBOC modulated signal. Then, a novel acquisition algorithm for CBOC modulated signal is proposed, named SSCM.

In the new algorithm SSCM, a special auxiliary signal for CBOC modulated signal is introduced to remove side peaks. Moreover, the data channel and pilot channel signals are utilized completely, to ensure the detection probability. Additionally, the subtraction of the correlation channels is used to suppress in-band noise. This way, the side peaks are removed before signal detection. And the acquisition precision will be improved, which accelerates the tracking process.

\section{Signal Model}

CBOC signal is recommended and produced by $\operatorname{BOC}(1,1)$ and $\mathrm{BOC}(6,1)$ signal. In Galileo E1 OS, CBOC signal has the same power for data and pilot channels. Two different implementations of CBOC are proposed for a $50 \% / 50 \%$ power split between data and pilot components. $\operatorname{CBOC}(6,1,1 / 11)$ is an important implementation method, where $1 / 11$ denotes the percentage of power of $\operatorname{BOC}(6,1)$ with respect to the total signal CBOC power. The PSD of $\operatorname{CBOC}(6,1,1 / 11)[13-16]$ is given by

$$
\begin{aligned}
G_{\mathrm{CBOC}(6,1,1 / 11)}(f)= & \frac{10}{11} G_{\mathrm{BOC}(1,1)}(f) \\
& +\frac{1}{11} G_{\mathrm{BOC}(6,1)}(f),
\end{aligned}
$$

where $G_{\mathrm{BOC}(1,1)}(f)$ and $G_{\mathrm{BOC}(6,1)}(f)$ can be represented with $G_{\mathrm{BOC}\left(f_{s}, f_{c}\right)}(f)$, which is the normalized baseband PSD of a BOC modulation, shown as below. From expression (2) of [17], $G_{\mathrm{BOC}\left(f_{\mathrm{s}}, f_{c}\right)}(f)$ can be expressed as below, when $2 f_{s} / f_{c}$ of $\operatorname{BOC}(1,1)$ and $\operatorname{BOC}(6,1)$ are both even.

$$
G_{\mathrm{BOC}\left(f_{s}, f_{c}\right)}(f)=f_{c}\left(\frac{\sin \left(\pi f / 2 f_{s}\right) \sin \left(\pi f / f_{c}\right)}{\pi f \cos \left(\pi f / 2 f_{s}\right)}\right)^{2} .
$$

The power spectrum and autocorrelation function of CBOC modulated signal are shown in Figures 1 and 2.

The subcarrier formula of $\operatorname{BOC}(1,1)$ and $\operatorname{BOC}(6,1)$ are given as follows:

$$
\begin{gathered}
\operatorname{SC}_{\mathrm{BOC}(1,1)}(t)= \begin{cases}\operatorname{sign}\left[\sin \left(\frac{2 \pi t}{T_{c}}\right)\right], & 0 \leq t \leq T_{c} \\
0, & \text { elsewhere, }\end{cases} \\
\operatorname{SC}_{\mathrm{BOC}(6,1)}(t)= \begin{cases}\operatorname{sign}\left[\sin \left(\frac{12 \pi t}{T_{c}}\right)\right], & 0 \leq t \leq T_{c} \\
0, & \text { elsewhere, }\end{cases}
\end{gathered}
$$

where $T_{c}$ is the code chip duration. Figure 3 shows the schematic diagram of $\operatorname{CBOC}(6,1,1 / 11)$ modulated signal. 


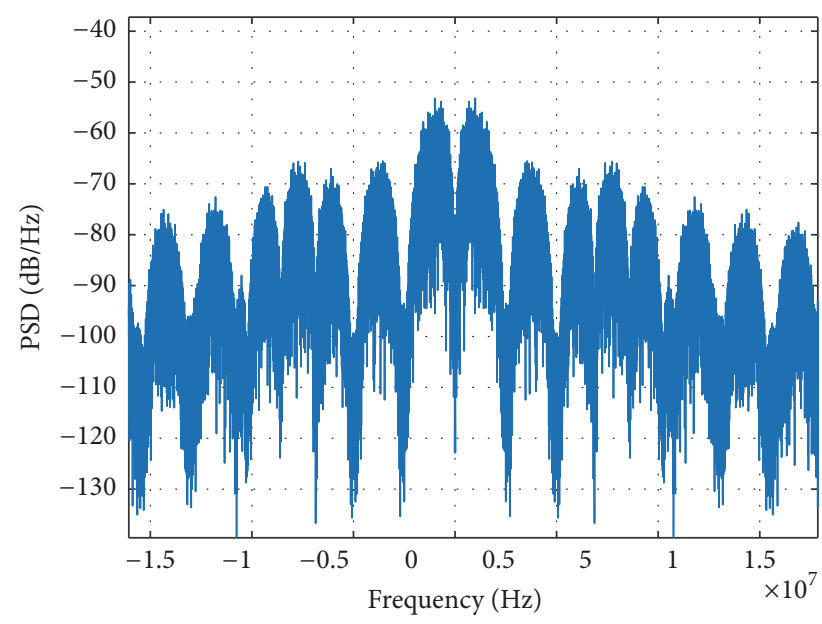

FIgURE 1: PSD of CBOC signal.

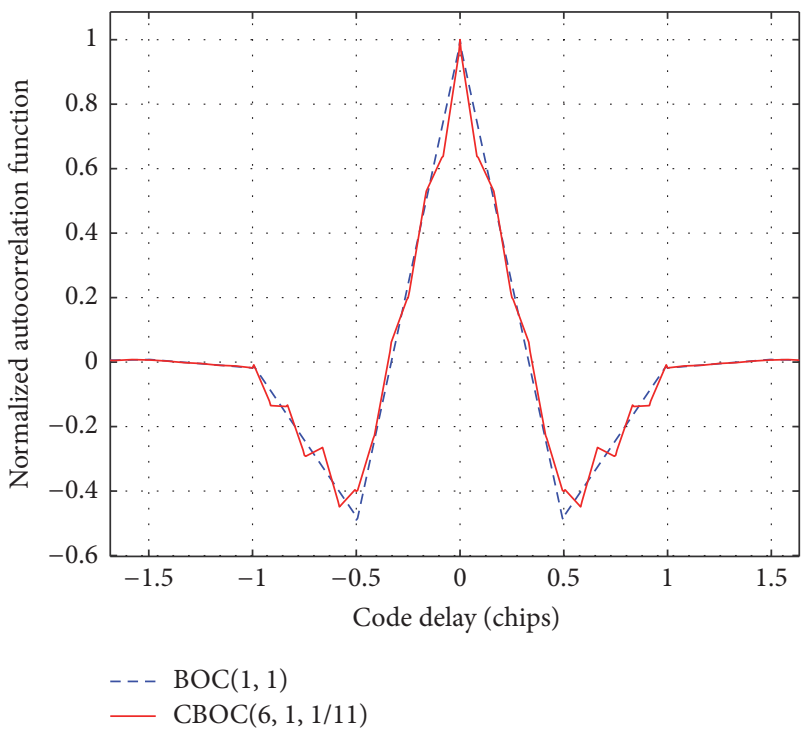

Figure 2: Normalized ACFs of $\operatorname{BOC}(1,1)$ and $\operatorname{CBOC}(6,1,1 / 11)$ signal.

\section{Proposed Acquisition Algorithm}

$\operatorname{CBOC}(6,1,1 / 11)$ is one of modulations for Galileo E1 signal [18], including E1-B and E1-C channels. $\operatorname{CBOC}(6,1,1 / 11)$ of baseband can be expressed mathematically as follows:

$$
\begin{aligned}
s(t)= & s_{\mathrm{E} 1-\mathrm{B}}(t)-s_{\mathrm{E} 1-\mathrm{C}}(t), \\
s_{\mathrm{E} 1-\mathrm{B}}(t)= & \left(\frac{1}{\sqrt{2}}\right) \cdot e_{\mathrm{E} 1-\mathrm{B}}(t) \\
& \cdot\left(P \cdot \mathrm{sc}_{\mathrm{E} 1-\mathrm{B}, a}(t)+\mathrm{Q} \cdot \mathrm{sc}_{\mathrm{E} 1-\mathrm{B}, b}(t)\right), \\
s_{\mathrm{E} 1-\mathrm{C}}(t)= & \left(\frac{1}{\sqrt{2}}\right) \cdot e_{\mathrm{E} 1-\mathrm{C}}(t) \\
& \cdot\left(P \cdot \mathrm{sc}_{\mathrm{E} 1-\mathrm{C}, a}(t)-\mathrm{Q} \cdot \mathrm{sc}_{\mathrm{E} 1-\mathrm{C}, b}(t)\right),
\end{aligned}
$$

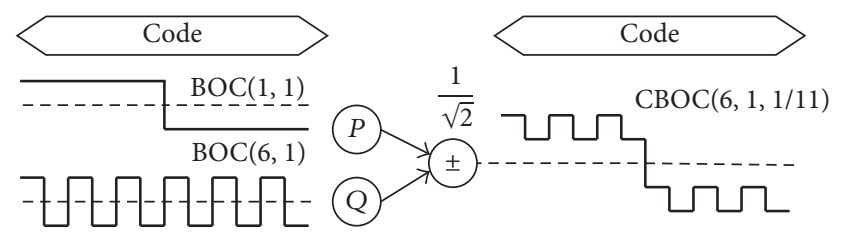

FIGURE 3: CBOC $(6,1,1 / 11)$ signal generation graph.

where $e_{\mathrm{E} 1-\mathrm{B}}(t)$ and $e_{\mathrm{E} 1-\mathrm{C}}(t)$ are the binary signal component of data and pilot at the code frequency $f_{c}\left(=1 / T_{c}\right)$. $P$ equals $\sqrt{10 / 11}$. $Q$ equals $\sqrt{1 / 11} . \mathrm{sc}_{\mathrm{E} 1-\mathrm{B}, a}(t), \mathrm{sc}_{\mathrm{E} 1-\mathrm{B}, b}(t), \mathrm{sc}_{\mathrm{E} 1-\mathrm{C}, a}(t)$, and $\operatorname{sc}_{\mathrm{E} 1-\mathrm{C}, b}(t)$, respectively, denote the subcarrier, which can be expressed as follows. And $f_{x}$ represents the subcarrier frequency

$$
\operatorname{sc}_{x}(t)=\operatorname{sign}\left(\sin \left(2 \pi f_{x} t\right)\right)
$$

For the case of a $\mathrm{CBOC}$ waveform on both data and pilot components, $\operatorname{CBOC}(6,1,1 / 11)$ and the rest of received signals of E1 signal can be expressed mathematically as follows:

$$
\begin{aligned}
& s_{r}(t)=A\left[c_{D}(t) d_{D}(t)\left(\operatorname{Psc}_{1}(t)+\operatorname{Qsc}_{6}(t)\right)\right. \\
& \left.-c_{P}(t)\left(\operatorname{Psc}_{1}(t)-\operatorname{Qsc}_{6}(t)\right)\right] \\
& \cdot \cos \left(2 \pi\left(f_{\mathrm{IF}}+f_{D}\right) t+\theta\right)+n(t),
\end{aligned}
$$

where $c_{D}$ and $c_{P}$ are the data and pilot channels spreading code sequences, $d$ is the navigation message, $\operatorname{BOC}(1,1)$ spreading symbols denoted $\mathrm{sc}_{1}(t)$ and $\operatorname{BOC}(6,1)$ spreading symbols denoted $\mathrm{sc}_{6}(t), A$ is the amplitude, $f_{\mathrm{IF}}$ and $f_{D}$ are IF frequency and Doppler frequency, respectively, $\theta$ is the unknown carrier phase, and $n(t)$ is the baseband equivalent noise of the received noise that is assumed to be Gaussian.

In order to introduce the method, the baseband signal of $\operatorname{CBOC}(6,1,1 / 11)$ is given by

$$
\begin{aligned}
& s(t)=A\left[c_{D}(t) d_{D}(t)\left(P \operatorname{sc}_{1}(t)+Q \operatorname{Qsc}_{6}(t)\right)\right. \\
& \left.-c_{P}(t)\left(P \operatorname{sc}_{1}(t)-\operatorname{Qsc}_{6}(t)\right)\right] .
\end{aligned}
$$

Based on the waveform of $\operatorname{CBOC}(6,1,1 / 11)$ signal, an auxiliary signal $a_{i}(t)(i=1,2)$ is introduced to signal acquisition process. The local reference signals of pilot and data channels with auxiliary signal are represented in the following equations.

$$
\begin{aligned}
& S_{D_{i}}(t)=C_{D-L}(t)\left(P \cdot \mathrm{SC}_{1}(t)+Q \cdot \mathrm{SC}_{6}(t)\right) a_{i}(t), \\
& S_{P_{i}}(t)=C_{P-L}(t)\left(P \cdot \mathrm{SC}_{1}(t)-Q \cdot \mathrm{SC}_{6}(t)\right) a_{i}(t), \\
& a_{i}(t)= \begin{cases}1.5, & (n-1) T_{c}<t<\left(n-\frac{2}{3}\right) T_{c} \\
0, & \left(n-\frac{2}{3}\right) T_{c}<t<\left(n-\frac{1}{3}\right) T_{c} \\
1.5 *(-1)^{i}, & \left(n-\frac{1}{3}\right) T_{c}<t<n T_{c}\end{cases}
\end{aligned}
$$

$$
i=1,2 \text {, }
$$


where $C_{D-L}(t)$ and $C_{P-L}(t)$ are local PRN code of data channel and pilot channel, $T_{c}$ is the PRN code interval, and $n$ is the number of $T_{c}$. Then the cross-correlation functions can be obtained, multiplying the local reference signals by $S_{\text {base }}(t)$. And $\tau$ represents code delay.

$$
\begin{aligned}
& R_{S / S_{D 1}}(\tau)=\sum_{-\infty}^{+\infty} S(t) S_{D_{1}}(t-\tau), \\
& R_{S / S_{D 2}}(\tau)=\sum_{-\infty}^{+\infty} S(t) S_{D_{2}}(t-\tau), \\
& R_{S / S_{P 1}}(\tau)=\sum_{-\infty}^{+\infty} S(t) S_{P_{1}}(t-\tau), \\
& R_{S / S_{P 2}}(\tau)=\sum_{-\infty}^{+\infty} S(t) S_{P_{2}}(t-\tau) .
\end{aligned}
$$

Then, the correlation results (13), (14), (15), and (16) can be computed based on equations (10), (11), and (12). $\operatorname{tri}(x / y)$ is a triangular function of width $2 y$, centred in $x=0$, where it has a unity value. It can be used to express the correlation peaks of correlation function.

$$
\left|R_{S / S_{D 1}}(\tau)\right|=\sum_{i=-11, i \neq 0}^{11} \alpha_{i} \cdot \operatorname{tri}\left(\frac{\tau+i / 12}{1 / 12}\right)
$$

where $1 / 12$ is the bottom width, decided by the subcarrier frequency of $\operatorname{BOC}(6,1)$. And the number of triangular functions is decided by the waveform of $S$ and $S_{D 1}$.

Based on the fixed component and ratio of $\operatorname{CBOC}(6,1,1 / 11), \alpha_{i}$ have fixed values through calculating. And the results of $\alpha_{i}$ are obtained as follows.

$$
\begin{aligned}
\alpha_{\mathrm{i}}= & {\left[\frac{1+2 \sqrt{10 / 11}}{8}, \frac{9}{44}, \frac{3+2 \sqrt{10 / 11}}{8}, \frac{9}{22}, \frac{1}{2}, \frac{9}{22},\right.} \\
& \frac{12-\sqrt{10}}{44}, \frac{1}{22}, \frac{7+2 \sqrt{10}}{88}, \frac{1}{4}, \frac{9}{88}, \frac{9}{88}, \frac{1}{4}, \frac{7+2 \sqrt{10}}{88}, \\
& \frac{1}{22}, \frac{12-\sqrt{10}}{44}, \frac{9}{22}, \frac{1}{2}, \frac{9}{22}, \frac{3+2 \sqrt{10 / 11}}{8}, \frac{9}{44}, \\
& \left.\frac{1+2 \sqrt{10 / 11}}{8}\right], \quad i=-11 \sim 11, i \neq 0 .
\end{aligned}
$$

Similarly, the correlation results $\left|R_{S / S_{D 2}}(\tau)\right|$ can be obtained.

$$
\begin{aligned}
& \left|R_{S / S_{D 2}}(\tau)\right|=\sum_{i=-11}^{11} \beta_{i} * \operatorname{tri}\left(\frac{\tau+i / 12}{1 / 12}\right), \\
& \beta_{i}=\left[\frac{1+2 \sqrt{10 / 11}}{8}, \frac{9}{44}, \frac{3+2 \sqrt{10 / 11}}{8}, \frac{9}{22}, \frac{1}{2}, \frac{9}{22},\right.
\end{aligned}
$$
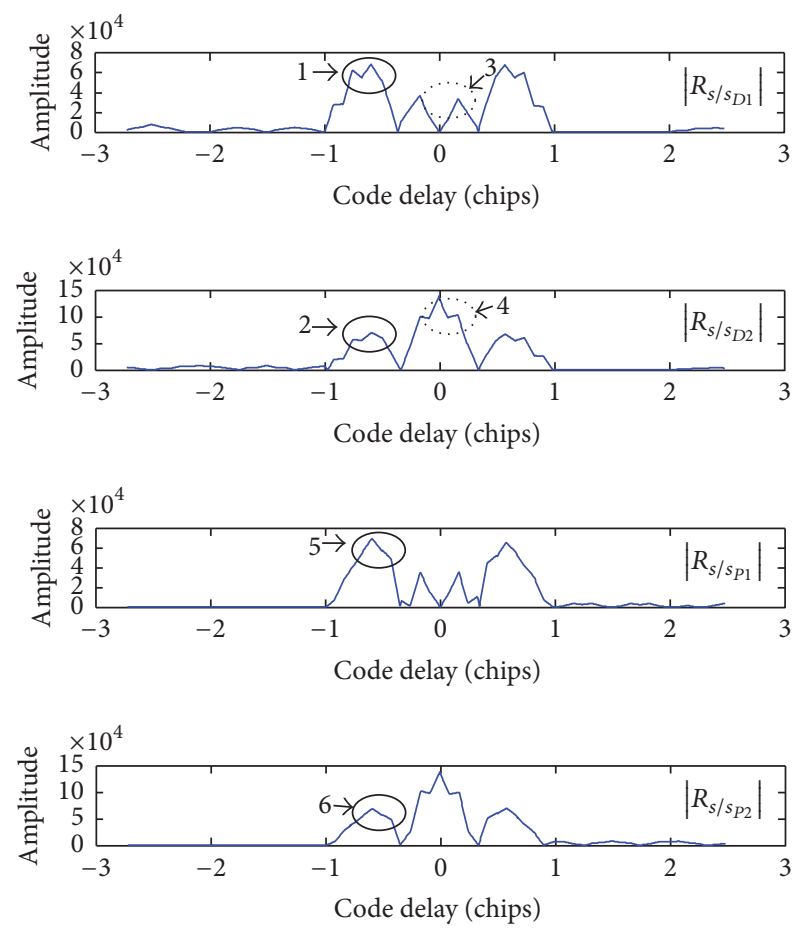

FIGURE 4: Correlation results of $\left|R_{S / S_{D 1}}(\tau)\right|,\left|R_{S / S_{D 2}}(\tau)\right|,\left|R_{S / S_{P 1}}(\tau)\right|$, and $\left|R_{S / S_{P 2}}(\tau)\right|$.

$$
\begin{aligned}
& \frac{12-\sqrt{10}}{44}, \frac{1}{22}, \frac{25+2 \sqrt{10}}{88}, \frac{3}{4}, \frac{63}{88}, 1, \frac{63}{88}, \frac{3}{4}, \\
& \frac{25+2 \sqrt{10}}{88}, \frac{1}{22}, \frac{12-\sqrt{10}}{44}, \frac{9}{22}, \frac{1}{2}, \frac{9}{22}, \frac{3+2 \sqrt{10 / 11}}{8}, \\
& \left.\frac{9}{44}, \frac{1+2 \sqrt{10 / 11}}{8}\right], \quad i=-11 \sim 11 .
\end{aligned}
$$

By calculating and comparing, $\left|R_{S / S_{D 1}}(\tau)\right|$ and $\left|R_{S / S_{D 2}}(\tau)\right|$ own similar side peaks, while there are seven differences, including $i=[-3,-2,-1,0,1,2,3]$. The same situation occurs on $\left|R_{S / S_{P_{1}}}(\tau)\right|$ and $\left|R_{S / S_{P_{2}}}(\tau)\right|$. All the correlation outputs have been simulated and shown in Figure 4.

Looking at Figure 4, we can find that label 1 and label 2 have the same shape as well as label 5 and label 6 . The result of the experiment is accordant with the theory. Then, if $\left|R_{S / S_{D 2}}(\tau)\right|$ is subtracted from $\left|R_{S / S_{D 1}}(\tau)\right|$, the side peaks will be removed nearly. Furthermore, when label 4 is subtracted from label 3 , the result is a main peak, restraining side peaks. The same situation occurs on $\left|R_{S / S_{P 1}}(\tau)\right|$ and $\left|R_{S / S_{P 2}}(\tau)\right|$. According to correlation results, outputs of data and pilot channels are obtained.

$$
\begin{aligned}
\left|R_{S / S_{D 2}}(\tau)\right|-\left|R_{S / S_{D 1}}(\tau)\right| & =\sum_{i=-3}^{3} \varepsilon_{i} \cdot \operatorname{tri}\left(\frac{\tau+i / 12}{1 / 12}\right), \\
\varepsilon_{i} & =\frac{9}{44}, \frac{1}{2}, \frac{27}{44}, 1, \frac{27}{44}, \frac{1}{2}, \frac{9}{44},
\end{aligned}
$$




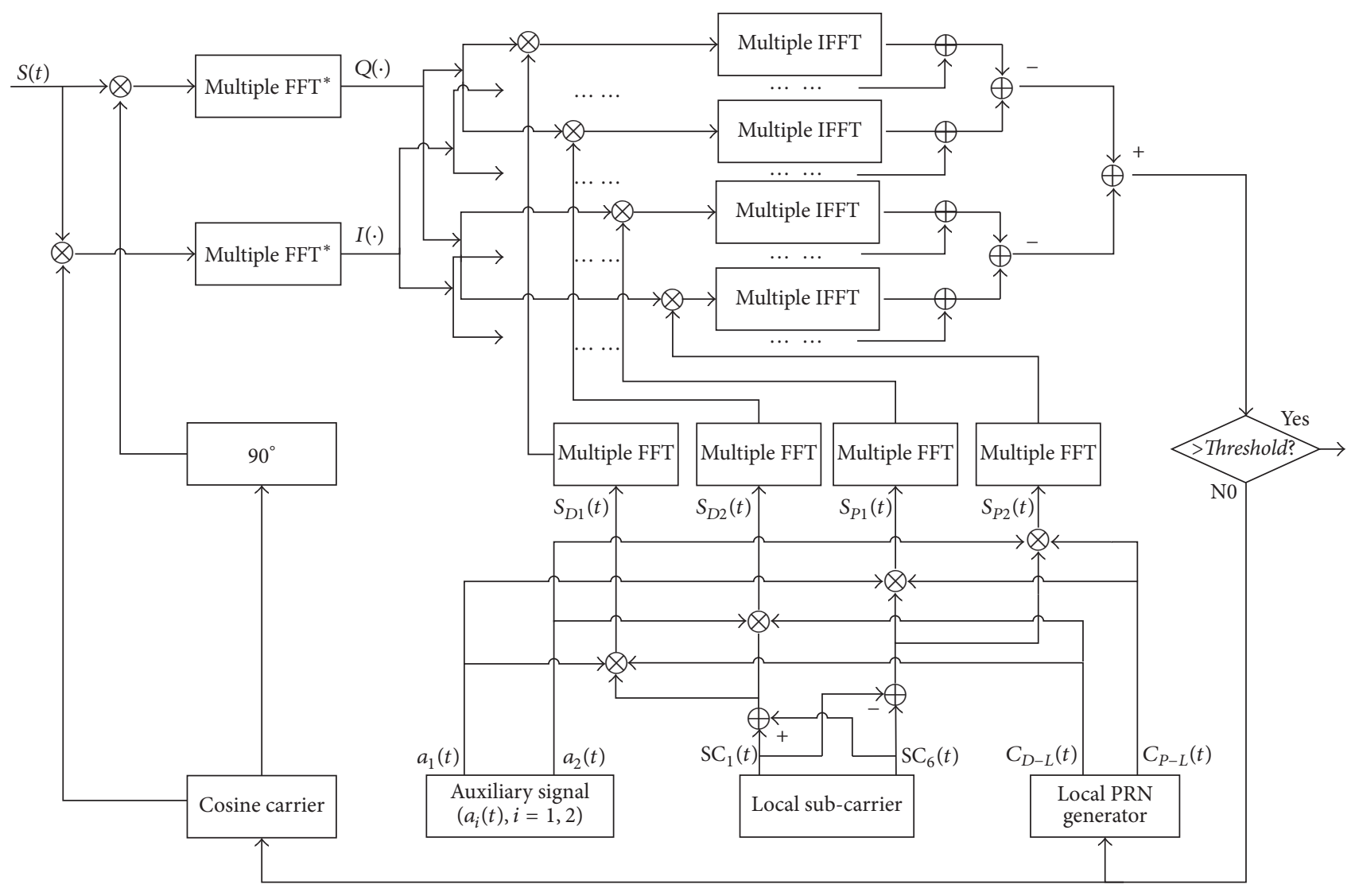

FIGURE 5: Architecture of SSCM algorithm.

$$
\begin{array}{r}
\left|R_{S / S_{P 2}}(\tau)\right|-\left|R_{S / S_{P 1}}(\tau)\right|=\sum_{i=-3}^{3} \varepsilon_{i} \cdot \operatorname{tri}\left(\frac{\tau+i / 12}{1 / 12}\right), \\
\varepsilon_{i}=\frac{9}{44}, \frac{1}{2}, \frac{27}{44}, 1, \frac{27}{44}, \frac{1}{2}, \frac{9}{44} .
\end{array}
$$

From (20) and (21), one can see that the correlation result is a linear superposition of several triangular functions, and the two functions are the same. Therefore, a normalized correlation function named special side-peak cancel method (SSCM) can be denoted as follows:

$$
\begin{aligned}
R_{\text {proposed }}(\tau)= & \left|R_{S / S_{D 2}}(\tau)\right|-\left|R_{S / S_{D 1}}(\tau)\right|+\left|R_{S / S_{P 2}}(\tau)\right| \\
& -\left|R_{S / S_{P 1}}(\tau)\right| \\
= & 2 \cdot \sum_{i=-3}^{3} \varepsilon_{i} \cdot \operatorname{tri}\left(\frac{\tau+i / 12}{1 / 12}\right) \\
\approx & 2 \cdot \operatorname{tri}\left(\frac{\tau}{1 / 3}\right) .
\end{aligned}
$$

From formula (22), the correlation function of SSCM contains only one main peak, solving the phase ambiguity problem effectively.

Based on subtract operation, the noise of each channel is restrained, while the main peak value of correlation output is uninfluenced. Furthermore, by using $\left|R_{S / S_{D 2}}(\tau)\right|-\left|R_{S / S_{D 1}}(\tau)\right|$ and $\left|R_{S / S_{P 2}}(\tau)\right|-\left|R_{S / S_{P 1}}(\tau)\right|$ in combination, a bigger main peak can be obtained, and the pilot channel and data channel are both fully used. Then the schematic diagram of SSCM algorithm is shown in Figure 5.

Eight correlators are employed in this architecture. And the in-phase branches are omitted to simplify structure.

In order to analyze detection probability $\left(P_{d}\right)$ and false alarm probability $\left(P_{f}\right)$, a detailed description of the spread spectrum signal acquisition theory [19] is used. In traditional acquisition scheme, the test criterion is given by

$$
T=\sum_{i=0}^{M-1} I_{i}^{2}+Q_{i}^{2}
$$

where $I_{i}$ and $Q_{i}$ are, respectively, in-phase and quadra-phase correlator outputs. $M$ is the number of noncoherent summations. Once the maximum correlation result is larger than a threshold, detection is declared. The main idea SSCM based is to construct local auxiliary signals, the cross-correlation of which with the received signal can be used to remove the undesired side peaks. We choose the test criterion as follows.

$$
\begin{aligned}
T & =\sum_{i=0}^{M-1}\left[\left(I_{S / S_{D 2}, i}^{2}+Q_{S / S_{D 2}, i}^{2}\right)-\left(I_{S / S_{D 1}, i}^{2}+Q_{S / S_{D 1}, i}^{2}\right)\right. \\
& \left.+\left(I_{S / S_{P 2}, i}^{2}+Q_{S / S_{P 2}, i}^{2}\right)-\left(I_{S / S_{P 1}, i}^{2}+Q_{S / S_{P 1}, i}^{2}\right)\right]
\end{aligned}
$$


where $I_{S / S_{D 2}, i}, Q_{S / S_{D 2}, i}, I_{S / S_{D 1}, i}, Q_{S / S_{D 1}, i}, I_{S / S_{P 2}, i}, Q_{S / S_{P 2}, i}, I_{S / S_{P 1}, i}$, and $Q_{S / S_{P 1}, i}$ are in-phase and quadra-phase correlate outputs when the local signals employ the auxiliary signal. All the outputs are shown in the following.

$$
\begin{aligned}
& I_{S / S_{D 1}, i} \\
& =\sqrt{\frac{T_{\text {sum }} C}{N_{0}}} \operatorname{sinc}\left(\Delta f T_{\text {sum }}\right) R_{S / S_{D 1}}(\Delta \tau) \cos (\Delta \varphi) \\
& +n_{I, i} \text {, } \\
& Q_{S / S_{D 1}, i} \\
& =\sqrt{\frac{T_{\text {sum }} C}{N_{0}}} \operatorname{sinc}\left(\Delta f T_{\text {sum }}\right) R_{S / S_{D 1}}(\Delta \tau) \sin (\Delta \varphi) \\
& +n_{\mathrm{Q}, i} \text {, } \\
& I_{S / S_{D 2}, i} \\
& =\sqrt{\frac{T_{\text {sum }} C}{N_{0}}} \operatorname{sinc}\left(\Delta f T_{\text {sum }}\right) R_{S / S_{D 2}}(\Delta \tau) \cos (\Delta \varphi) \\
& +n_{I, i} \\
& Q_{S / S_{D 2}, i} \\
& =\sqrt{\frac{T_{\text {sum }} C}{N_{0}}} \operatorname{sinc}\left(\Delta f T_{\text {sum }}\right) R_{S / S_{D 2}}(\Delta \tau) \sin (\Delta \varphi) \\
& +n_{\mathrm{Q}, i} \text {, } \\
& I_{S / S_{P 1}, i} \\
& =\sqrt{\frac{T_{\text {sum }} C}{N_{0}}} \operatorname{sinc}\left(\Delta f T_{\text {sum }}\right) R_{S / S_{P 1}}(\Delta \tau) \cos (\Delta \varphi) \\
& +n_{I, i}, \\
& Q_{S / S_{P 1}, i} \\
& =\sqrt{\frac{T_{\text {sum }} C}{N_{0}}} \operatorname{sinc}\left(\Delta f T_{\text {sum }}\right) R_{S / S_{P 1}}(\Delta \tau) \sin (\Delta \varphi) \\
& +n_{\mathrm{Q}, i} \text {, } \\
& I_{S / S_{P 2}, i} \\
& =\sqrt{\frac{T_{\text {sum }} C}{N_{0}}} \operatorname{sinc}\left(\Delta f T_{\text {sum }}\right) R_{S / S_{P 2}}(\Delta \tau) \cos (\Delta \varphi) \\
& +n_{I, i} \text {, } \\
& Q_{S / S_{P 2}, i} \\
& =\sqrt{\frac{T_{\text {sum }} C}{N_{0}}} \operatorname{sinc}\left(\Delta f T_{\text {sum }}\right) R_{S / S_{P 2}}(\Delta \tau) \sin (\Delta \varphi) \\
& +n_{\mathrm{Q}, i} \text {. }
\end{aligned}
$$

Here $\operatorname{sinc}(x)$ is equal to $\sin (x) / x . \Delta \tau$ means the code phase error, $T_{\text {sum }}$, is the coherent integration time, $\Delta f$ represents the Doppler frequency, $C / N_{0}$ means the ratio of carrier and noise, and $\Delta \varphi$ refer to the frequency wipe-off error. $n_{I, i}$ and $n_{\mathrm{Q}, i}$ are the correlation calculation results of an additive white Gaussian noise with zero and single-sided noise PSD $N_{0}$.

Then the four terms $\sum_{i=0}^{M-1}\left(I_{S / S_{D 2}, i}^{2}+Q_{S / S_{D 2}, i}^{2}\right)$, $\sum_{i=0}^{M-1}\left(I_{S / S_{D 1}, i}^{2}+Q_{S / S_{D 1}, i}^{2}\right), \quad \sum_{i=0}^{M-1}\left(I_{S / S_{P 2}, i}^{2}+Q_{S / S_{P 2}, i}^{2}\right), \quad$ and $\sum_{i=0}^{M-1}\left(I_{S / S_{P 1}, i}^{2}+Q_{S / S_{P 1}, i}^{2}\right)$ follow $\chi^{2}$ distribution with $2 M$ degrees of freedom (DOF), in which the noncentrality parameters of the four terms are

$$
\begin{aligned}
& a_{1}^{2}=M \cdot T_{\text {sum }} \cdot \frac{C}{N_{0}} \cdot \operatorname{sinc}^{2}\left(\Delta f T_{\text {sum }}\right) \cdot R_{S / S_{D 2}}^{2}(\Delta \tau), \\
& a_{2}^{2}=M \cdot T_{\text {sum }} \cdot \frac{C}{N_{0}} \cdot \operatorname{sinc}^{2}\left(\Delta f T_{\text {sum }}\right) \cdot R_{S / S_{D 1}}^{2}(\Delta \tau), \\
& a_{3}^{2}=M \cdot T_{\text {sum }} \cdot \frac{C}{N_{0}} \cdot \operatorname{sinc}^{2}\left(\Delta f T_{\text {sum }}\right) \cdot R_{S / S_{P 2}}^{2}(\Delta \tau), \\
& a_{4}^{2}=M \cdot T_{\text {sum }} \cdot \frac{C}{N_{0}} \cdot \operatorname{sinc}^{2}\left(\Delta f T_{\text {sum }}\right) \cdot R_{S / S_{P 1}}^{2}(\Delta \tau) .
\end{aligned}
$$

And the test criterion without noise can be expressed as

$$
\begin{aligned}
T & \approx M \cdot T_{\text {sum }} \cdot \frac{C}{N_{0}} \cdot \operatorname{sinc}^{2}\left(\pi \Delta f T_{\text {sum }}\right) \cdot\left(R_{S / S_{D 2}}^{2}(\Delta \tau)\right. \\
& \left.-R_{S / S_{D 1}}^{2}(\Delta \tau)+R_{S / S_{P 2}}^{2}(\Delta \tau)-R_{S / S_{P 1}}^{2}(\Delta \tau)\right)=a_{1}^{2} \\
& -a_{2}^{2}+a_{3}^{2}-a_{4}^{2} .
\end{aligned}
$$

According to the statistical theory [19-21], in order to simplify the computation of theoretical $P_{f a}$ and $P_{d}$, the detection probability and false alarm probability of the proposed method have similar form as the GRASS technique [10]. And the Gaussian Q-function is adopted to approximate

$$
P_{d}(V) \approx Q\left(\frac{V-4 M-M(T-4)}{4 \sqrt{M(4+T)}}\right),
$$

where $V$ denotes threshold of false alarm, which can be calculated as

$$
P_{f a}(V)=\exp \left(-\frac{V}{2}\right) \sum_{n=0}^{M-1} \frac{1}{n !}\left(\frac{V}{2}\right)^{n} .
$$

\section{Simulation and Analysis}

In order to verify the effectiveness and validity of SSCM, it is necessary to analyze the efficiency in terms of correlation function, peak-to-average ratio (PAR) with different noise, detection probability $\left(P_{d}\right)$, false alarm probability $\left(P_{f}\right)$, and processing complexity. In the following comparisons, we assume the following parameters: the center frequency $f_{c}$ is $48 \mathrm{MHz}$, sampling frequency $f_{s}$ is $192 \mathrm{MHz}$, accumulation time $T$ is $1 \mathrm{~ms}$, and signal-to-noise rate (SNR) range is $-35 \sim$ $0 \mathrm{~dB}$. 


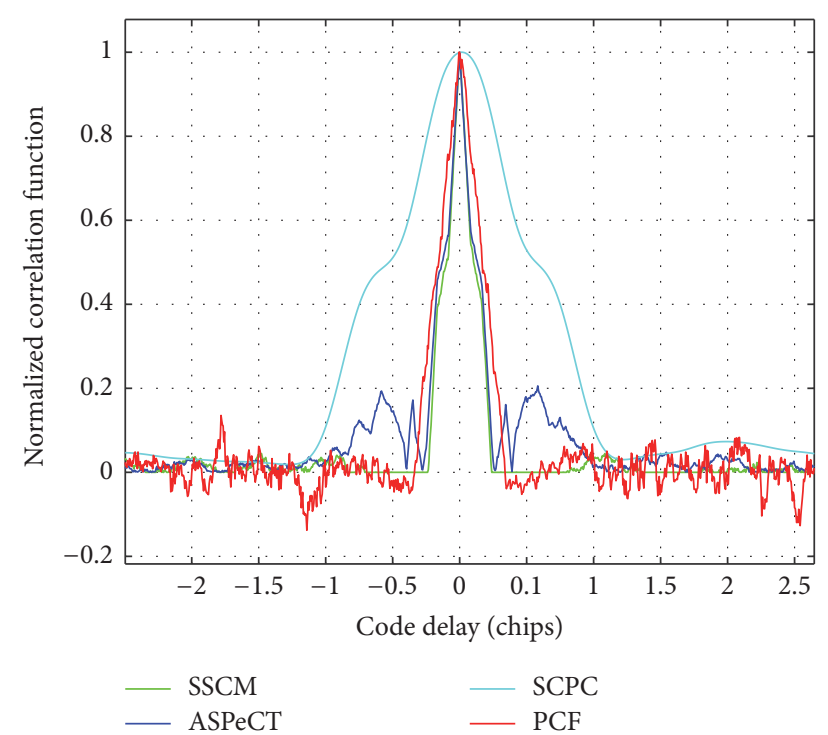

Figure 6: Normalized correlation functions of SSCM, ASPeCT, SCPC, and PCF.

Figure 6 shows the normalized correlation functions of these four methods. And the SNR is set at $-15 \mathrm{~dB}$. SCPC method is similar to that of BPSK, and this method destroys the narrow peak of autocorrelation function (ACF). Therefore, SCPC method is not suitable to CBOC signal. For ASPeCT method, the influence of remaining side peaks still exist. With PRN code delay and Doppler shift, each value is tested in order to get a correlative result. Once the maximum correlation result exceeds the setting threshold, detection will occur. It is probably that one of side peak's energy will be larger than the main peak under the influence of remaining side peaks; thus false acquisition will happen, and this side peak will be locked. In these four methods, PCF method has similar ACF with SSCM method, owning only one main peak. Nevertheless, the two methods differed in power efficiency. From formula (4) one can find that CBOC signal owns data component and pilot component. In PCF method, only one component is utilized, while the two components are fully used in SSCM method. And the main peak of SSCM will be higher than PCF's. Through comparison and analysis, it is found that PCF method owns the best correlation function among PCF, ASPeCT, and SCPC, which is similar to SSCM. Then, take PCF method as example, the performances of PCF and SSCM are compared. In Figure 7, the nonnormalized correlation functions of PCF and SSCM method are shown.

In $\operatorname{CBOC}(6,1,1 / 11)$ modulation, data component and pilot component are modulated in the same carrier. And both components have the same Doppler shift and code synchronous message. Therefore, depending on using a combination of the two components, PAR of SSCM method is increased to guarantee $P_{d}$ under severe environment.

Along with SNR increasing from $-35 \mathrm{~dB}$ to $0 \mathrm{~dB}$, simulation shows that SSCM method own the best PAR than other three methods. From formula (20), $\left|R_{S / S_{D 2}}(\tau)\right|-\left|R_{S / S_{D 1}}(\tau)\right|$ and $\left|R_{S / S_{P 2}}(\tau)\right|-\left|R_{S / S_{P 1}}(\tau)\right|$ can suppress the noise of each component, which contribute to PAR. And SCPC owns the

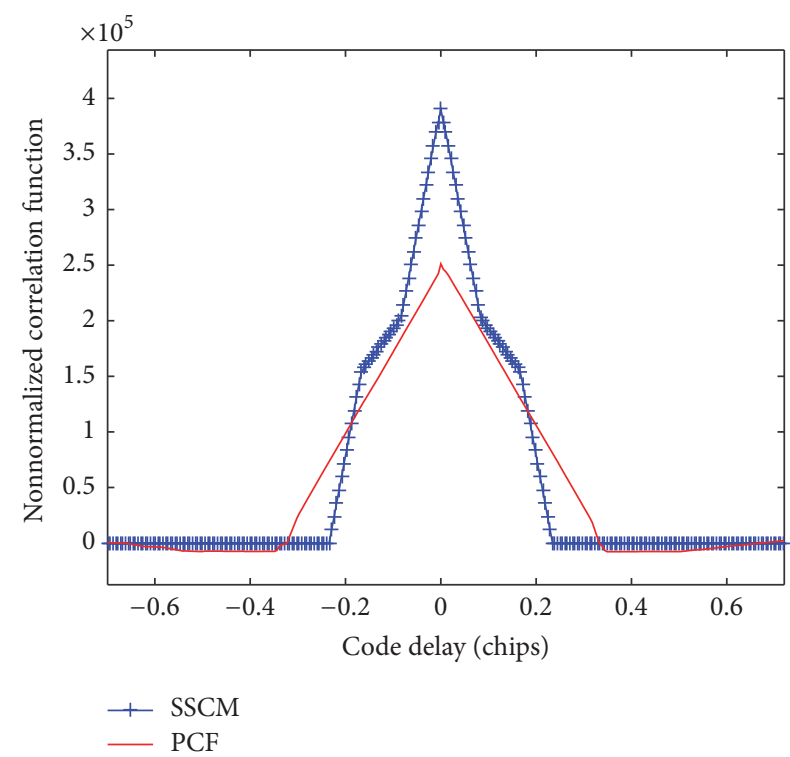

FIGURE 7: Nonnormalized correlation functions of SSCM and PCF.

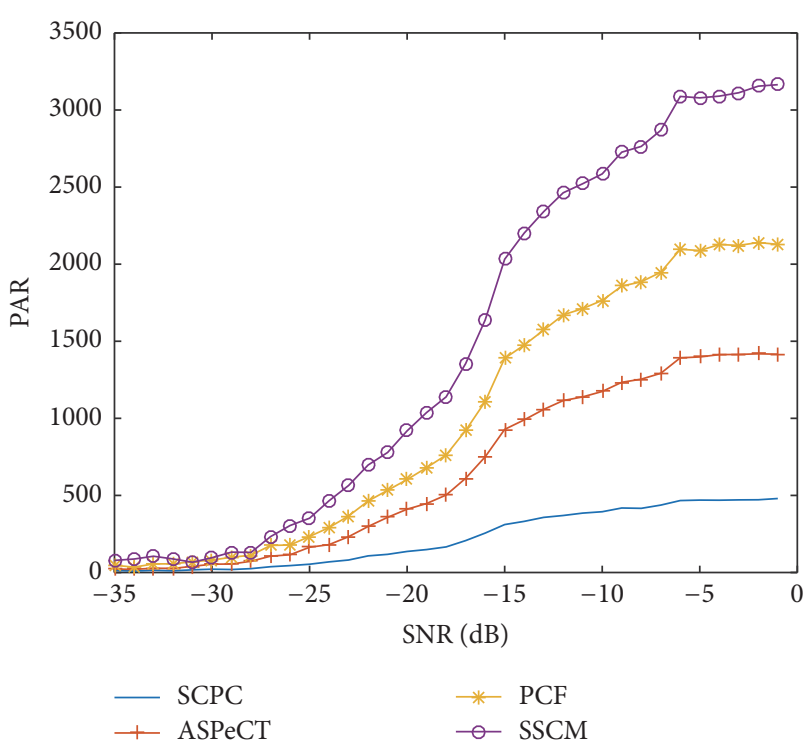

FIGURE 8: PARs of four methods.

worst PAR due to its combination of in-phase correlation output and quadra-phase correlation output, which cause channel noise more severe. PARs of these four methods are shown in Figure 8.

With a fixed false alarm probability $P_{f a}=10^{-5}$, the performance of the SSCM and PCF acquisition method is also shown in the following for comparison.

The detection probabilities of both proposed technique and PCF method are drawn in Figure 9 with different $T_{p}$ and $M$, under the assumption of fixed false alarm probability. And the detection probabilities increase with $C / N_{0}, T_{p}$ and $M$ growth. And the acquisition performance of SSCM method increases 1 to $2 \mathrm{~dB}$ compared with the PCF method. The 


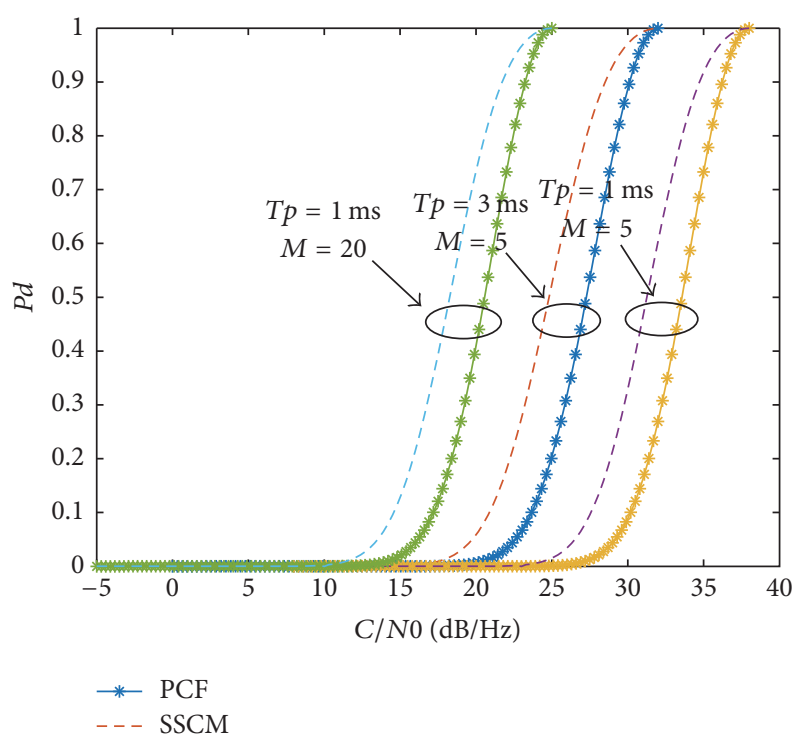

FIgURE 9: Detection probability for CBOC, $P_{f a}=10^{-5}$.

reason for this phenomenon is that the proposed method uses data channel and pilot channel completely to get the correlation peak. Moreover, the proposed method has no threat with acquisition ambiguity problems. So the proposed scheme is robust compared with PCF scheme. Another characteristic of the proposed unambiguous methods is that it has a narrower peak. It can provide sufficiently small code delay to insure the acquisition precision.

\section{Conclusion}

In this paper, the principle and characteristics of $\operatorname{CBOC}(6,1,1 / 11)$ are studied. And a new acquisition method for $\operatorname{CBOC}(6,1,1 / 11)$ modulation is proposed, which can remove the ambiguity threat. Eight correlators are used in this method and result in complexity of acquisition structure increasing. However, the acquisition technique can remove side peaks completely, meaning that the ambiguity problem can be restrained. Moreover, SSCM method is suitable for working in a serious condition. And the detection probability of CBOC can be guaranteed, which devote to average acquisition time.

\section{Conflicts of Interest}

The authors declare that they have no conflicts of interest.

\section{Acknowledgments}

This work was supported by the National Natural Science Foundation of China (no. 61501309), the Program for Liaoning Innovative Research Team in University (no. LT2011005), New Century Program for Excellent Talents of Ministry of Education of China (no. NCET-11-1013), Project of Science and Technology Department of Liaoning Province (no. 20121038), and Project of Education Department of Liaoning Province (no. L2013085).

\section{References}

[1] S. Tom, "Perspective on GNSS compatibility and interoperability," in Second meeting of the International Committee on Global Navigation Satellite System, pp. 1025-1056, Bangalore, India, 2007.

[2] G. W. Hein, J.-A. Avila-Rodriguez, S. Wallner et al., "MBOC: the new optimized spreading modulation recommended for GALILEO L1 OS and GPS L1C," in Proceedings of the 2006 IEEE/ION Position, Location, and Navigation Symposium, pp. 883-892, USA, April 2006.

[3] N. Martin, V. Leblond, G. Guillotel, and V. Heiries, "BOC(x, y) signal acquisition techniques and performances," in Proceeding of U.S. Institute of Navigation GPS/GNSS Conference, pp. 188198, Portland, 2003.

[4] O. Julien, C. Macabiau, M. E. Cannon, and G. Lachapelle, "ASPeCT: unambiguous sine-BOC(n,n) acquisition/tracking technique for navigation applications," IEEE Transactions on Aerospace and Electronic Systems, vol. 43, no. 1, pp. 150-162, 2007.

[5] J. Ren, G. Yang, W. Jia, and M. Yao, "Unambiguous tracking method based on combined correlation functions for sine/cosine-BOC CBOC and ALTBOC modulated signals," Radioengineering, vol. 23, no. 1, pp. 244-253, 2014.

[6] Z. Yao, "A new unambiguous tracking technique for sine$\operatorname{BOC}(2 \mathrm{n}, \mathrm{n})$ signals," in Proceedings of the International Technical meeting of the Satellite Division, ION GNSS 21st, pp. 16-19, Savannah, GA, USA, 2008.

[7] Z. Yao, M. Lu, and Z. Feng, "Unambiguous technique for multiplexed binary offset carrier modulated signals tracking," IEEE Signal Processing Letters, vol. 16, no. 7, pp. 608-611, 2009.

[8] V. Heiries, J.-À. Àvila-Rodriguez, M. Irsigler, G. W. Hein, E. Rebeyrol, and D. Roviras, "Acquisition performance analysis of composite signals for the LI OS optimized signal," in Proceedings of the 18th International Technical Meeting of the Satellite Division of The Institute of Navigation, ION GNSS 2005, pp. 877889, usa, September 2005.

[9] Y. Lee, D. Chong, I. Song, S. Y. Kim, G.-I. Jee, and S. Yoon, "Cancellation of correlation side-peaks for unambiguous BOC signal tracking," IEEE Communications Letters, vol. 16, no. 5, pp. 569-572, 2012.

[10] Z. Yao, M. Lu, and Z. Feng, "Unambiguous sine-phased binary offset carrier modulated signal acquisition technique," IEEE Transactions on Wireless Communications, vol. 9, no. 2, pp. 577580, 2010.

[11] F. Shen, G. Xu, and D. Xu, "Unambiguous acquisition technique for cosine-phased binary offset carrier signal," IEEE Communications Letters, vol. 18, no. 10, pp. 1751-1754, 2014.

[12] Y. Zhou, X. Hu, T. Ke, and Z. Tang, "Ambiguity mitigating technique for cosine-phased binary offset carrier signal," IEEE Transactions on Wireless Communications, vol. 11, no. 6, pp. 1981-1984, 2012.

[13] J. A. Avila-Rodriguez, "Revised combined GALILEO/GPS frequency and signal performance analysis," in Proceedings of ION GNSS, pp. 13-16, Long Beach, Calif, USA, 2005.

[14] M. Flissi, K. Rouabah, D. Chikouche, A. Mayouf, and S. Atia, "Performance of new BOC-AW-modulated signals for GNSS system," EURASIP Journal on Wireless Communications and Networking, vol. 2013, no. 1, article no. 124, 2013.

[15] D. W. Lim, D. J. Cho, H. H. Choi, and S. J. Lee, "A simple and efficient code discriminator for a MBOC signal tracking," IEEE Communications Letters, vol. 17, no. 6, pp. 1088-1091, 2013. 
[16] J. A. Avila-Rodriguez, S. Wallner, G. W. Hein, E. Rebeyrol, and O. Julien, "CBOC: an implementation of MBOC. CNES-ESA," in Proceedings of the 1st Workshop on GALILEO Signals and Signal Processing, Toulouse, France, 2006.

[17] J. W. Betz, "Binary offset carrier modulations for radionavigation," NAVIGATION: Journal of the Institute of Navigation, vol. 48, no. 4, pp. 227-246, 2001.

[18] European Union, European GNSS (Galileo) Open Service, Signal in Space Interface Control Document, SIS ICD, Issue 1.2, November, 2015.

[19] F. Bastide, O. Julien, C. Macabiau, and B. Roturier, "Analysis of L5/E5 acquisition, tracking and data demodulation thresholds," in Proceedings of the U.S. Institute of Navigation GPS Conference, pp. 2196-2207, Portland, Ore, USA, 2002.

[20] J. Proakis and M. Salehi, Digital Communications, McGraw-Hill, 2001.

[21] H. Van Trees, Detection, Estimation And Modulation Theory, Chapter 5, Wiley-Interscience, 2001. 


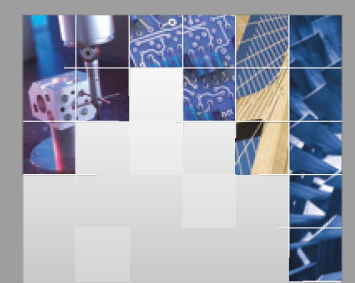

\section{Enfincering}
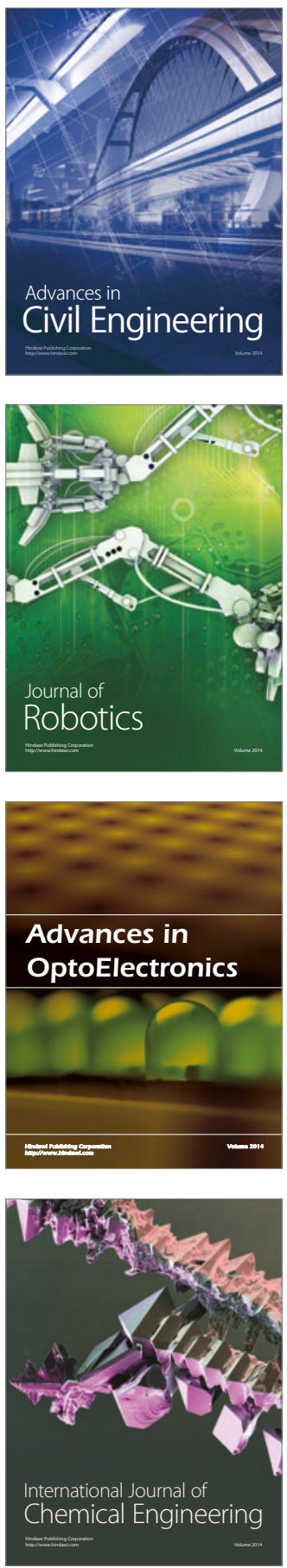

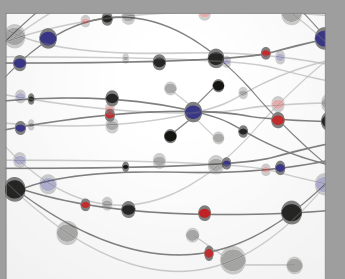

The Scientific World Journal

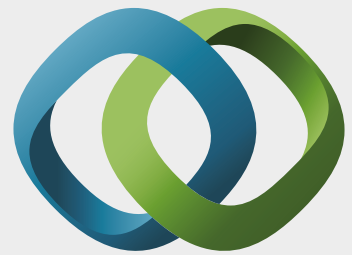

\section{Hindawi}

Submit your manuscripts at

https://www.hindawi.com
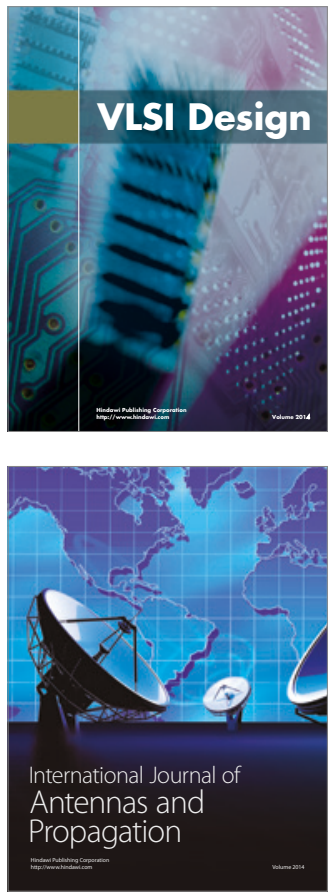

\section{Rotating}

Machinery
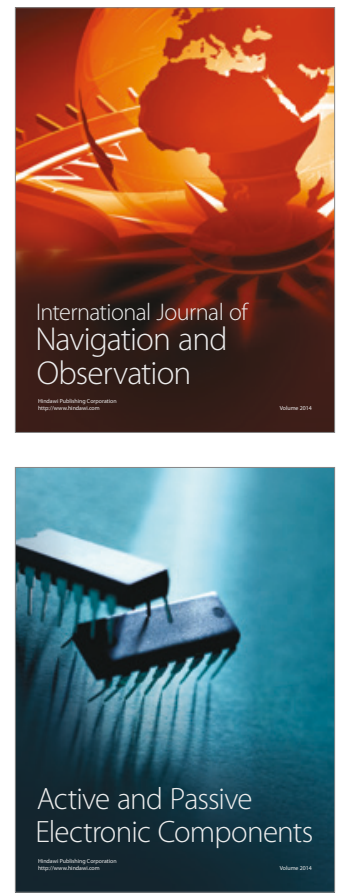
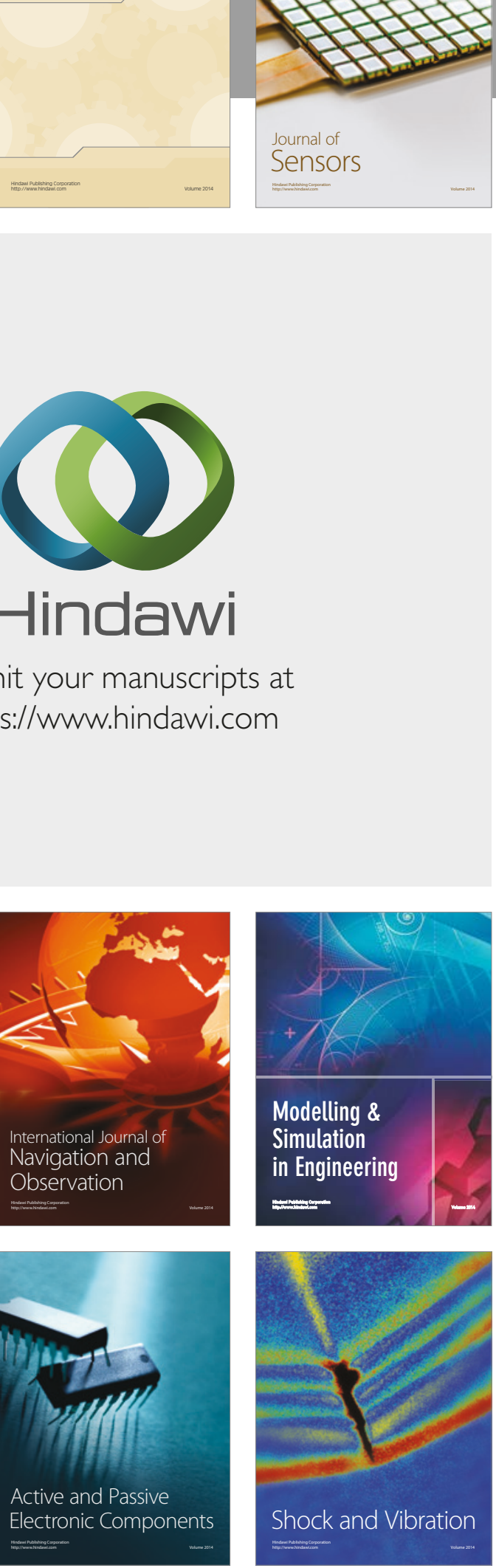
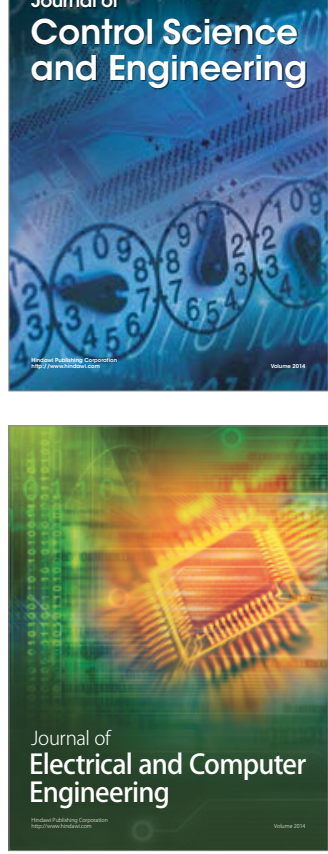

Distributed

Journal of

Control Science

and Engineering
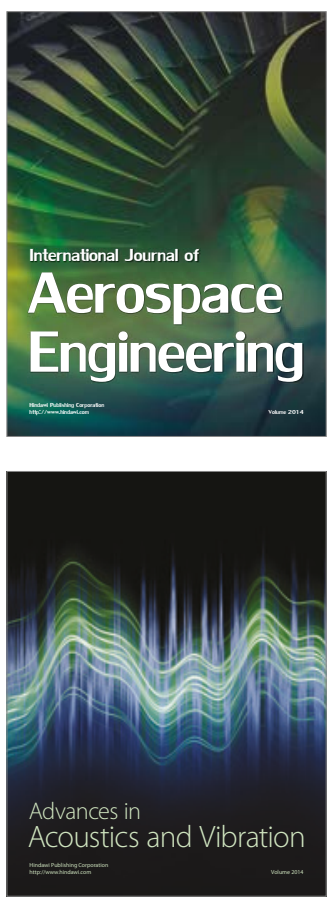

Sensor Networks 